\title{
Generalized Method for Constructing Magic Cube by Folded Magic Squares
}

\author{
Omar A. Dawood ${ }^{1}$ \\ Ph.D. Student at Computer Science Department, University of Technology, Baghdad, Iraq \\ Email: The_lionofclub@yahoo.com
}

\author{
Abdul Monem S. Rahma ${ }^{2}$, Abdul Mohssen J. Abdul Hossen ${ }^{3}$ \\ Computer Science Department, University of Technology, Baghdad, Iraq \\ Email: \{Monem.rahma, Abdulmoohsen53\}@yahoo.com
}

\begin{abstract}
In the present paper we have developed a new method for constructing magic cube by using the folded magic square technique. The proposed method considers a new step towards the magic cube construction that applied a good insight and provides an easy generalized technique. This method generalized the design of magic cube with $\mathrm{N}$ order regardless the type of magic square whether odd order, singly even order or doubly even order. The proposed method is fairly easy, since it have depended mainly on the magic square construction methods, and all what the designer need is just how to builds six magic square sequentially or with constant difference value between each pair of the numbers in the square matrix, whereby each one of this magic square will represents the surface or dimension for magic cube configuration. The next step for the designer will be how to arrange each square in the proper order to constitute the regular cube in order to maintain the properties of magic cube, where the sum of rows, columns and the diagonals from all directions are the same.
\end{abstract}

Index Terms-Magic Square, Magic Cube, Magic Constant, Magic Sum, Folded Square.

\section{INTRODUCTION}

Magic squares have different beliefs according to each country and population. They were found in disparate cultures and spread widely in several civilizations. Ancient China, Greek, Egypt, India, Europe and Mesopotamian whose has been studied magic square for at least three thousand years. Magical squares remain an interesting phenomenon to study, both mathematically and historically. It is equivalent to a square matrix as a painting full of numbers or letters in certain arrangements, from the mathematical view it is the most interesting in computational squares which consists of $n^{2}$ boxes, called cells or boxes, filled with different integers [1]. This array is called magic square of $\mathrm{n} \times \mathrm{n}$ numbers containing the numbers with consecutive order as $1 ; 2 \ldots \mathrm{n}^{2}$. The total elements in any row, column, or diagonals should be the same. Magic square may be divided into parts with letters, symbols, talisman, discrete words or numbers that are engraved or written in it, whether gathered vertically, horizontally or diagonally, forming the same amount or may be added to spell out the same words that called alpha magic squares. In out dated times it was believed that such squares have magical properties, and perhaps in touch with the orbit and the stars in addition to solar system. Figuration of magic square is computationally sensitive, and the indicator of rows and columns influenced by restrictions, and also affected some diagonals constraints. Moving one indicator affects at least of one row, one column value and the diagonals values. Thus, swap two values will change all the possible solutions for all the other indices [2].

The extension of magic square to more dimensions gives a magic cube. The magic cube likes the magic square from the point of probability construction that increases dramatically with the order of magic cube [3]. The magic constant (MC), magic vector or magic number, these terms are synonyms that can be calculated by the derivative formula (1).

$$
M C=\frac{n\left(n^{2}+1\right)}{2}
$$

Thus, $3 * 3$ normal magic square must have its rows, columns and diagonals adding to $\mathrm{MC}=15$

$$
M C=\frac{3\left(3^{2}+1\right)}{2}=\frac{30}{2}=15,4 * 4 \text { to } \mathrm{MC}=34,5 * 5
$$

to $\mathrm{MC}=65$ and $8 * 8$ to $\mathrm{MC}=260$, and so on. The Magic Sum (MS) is another significant term that includes the summation to the all numbers by (rows, columns and diagonals) in a magic square uses the following formula (2).

$$
M S=\frac{n^{2}\left(n^{2}+1\right)}{2}
$$

The MS for $3 * 3=45$, MS for $4 * 4=136$, MS for $5 * 5=325$, MS for $8 * 8=2080$ and so on, other method for calculating MS is by multiplying the MC by dimension of the magic square [4]. The following Fig. 1 below is a simple example of a magic square of order 3 with 9 values arranged consecutively in the magical order. So, suppose $\mathrm{MC}$ is the number that each row, column and 
diagonal must be add up to a vector numbers, and $(\mathrm{P})$ is a pivot element for the numbers through which the magic square is determined and constructed. The pivot element in the magic square represents the center element in the middle square as it explained and mentioned with shaded central number, and through which can be determined some properties of the magic square.

\begin{tabular}{|c|c|c|}
\hline 8 & 1 & 6 \\
\hline 3 & 5 & 7 \\
\hline 4 & 9 & 2 \\
\hline
\end{tabular}

Fig.1. Magic Square of Order 3

The pivot element at any magic square of odd order with sequential numbers can be calculated with the following formula:

$$
P=\frac{n^{2}+1}{2}
$$

For example: the following two examples include pivot computing in magic square of order three and order five.

$$
P=\frac{n^{2}+1}{2}=\frac{3^{2}+1}{2}=5, P=\frac{n^{2}+1}{2}=\frac{5^{2}+1}{2}=13
$$

and so on Here, is another formula to determine the pivot element in non-sequential odd order numbers that might begin with indeterminate integer number, or have a period, in another word that have difference between the numbers greater than one. As stated below in equation (4). Where $\mathrm{N}=$ square order, $\mathrm{A}=$ starting number and $\mathrm{D}=$ difference number that represents the difference between the successive and the previous numbers. The Fig. 4 below states three of different examples a, b and c respectively that explains the whole notation [5][6].

$$
P=\left(\frac{2 A+D\left(N^{2}-1\right)}{2}\right)
$$

\begin{tabular}{|l|l|l|}
\hline 75 & 54 & 69 \\
\hline 60 & 66 & 72 \\
\hline 63 & 78 & 57 \\
\hline
\end{tabular}

(a) : $\mathrm{N}=3, \mathrm{~A}=54$ and

\begin{tabular}{|l|l|l|}
\hline 52 & 17 & 42 \\
\hline 27 & 37 & 47 \\
\hline 32 & 57 & 22 \\
\hline
\end{tabular}

(b) : $N=3, A=17$ and $D=5$

\begin{tabular}{|c|c|c|}
\hline 17 & 3 & 13 \\
\hline 7 & 11 & 15 \\
\hline 9 & 19 & 5 \\
\hline
\end{tabular}

(c) : $N=3, A=3$ and $D=2$
Fig.2. Three Magic Square of Order 3 with constant difference

$$
\begin{aligned}
& \text { (a) } P=\left(\frac{2 * 54+3\left(3^{2}-1\right)}{2}\right)=66 \\
& \text { (b) } P=\left(\frac{2 * 17+5\left(3^{2}-1\right)}{2}\right)=37
\end{aligned}
$$

$$
\text { (c) } P=\left(\frac{2 * 3+2\left(3^{2}-1\right)}{2}\right)=11
$$

\section{RELATED WORKS}

For long years, several researchers and mathematician specialists have created many related works about the magic square and magic cube. The following are some studies and discussions to the prior labor which so far associated work to the proposed method: In 1970, Richard Meyers invented a perfect eighth-order magic cube that is known by his name Meyers cube. The Meyers cube consist of sequence of numbers from 1 to $\mathrm{n}^{3}$ that arranged in each row, column and diagonal with cross section way. The Meyers cube is interested in several symmetries properties which assume that the cube is associative and every orthogonal and diagonal line sum to the same specific number. The corners values in the inner small cube as well as the corners values of each rectangular in the Meyers cube also sum to a constant certain number. The prominent feature for the symmetries properties makes that possible for a tantalizing number and for rearrangements of the cube [7].

In 1981 J. Barkley Rosser and Robert J. Walker are another two researchers whom introduced a new approach for constructing a perfect eighth-order magic cube. They also explained and proved that the perfect pan diagonal cubes are found for whole the orders with multiples of 8 and also for all the odd orders that more than 8 order. This approach considers a generalized method for the magic cube with order of multiple eight [8]. In 1988, John Hendricks submitted new ideas and published many refereed related papers. So he developed a simple and clear technique in constructing an odd order magic cube with $\mathrm{N}$ order. As well he published an extended dimension of hyper cubes with four, five and six dimensions; in addition, he had an elegant works and great contribution in the magic square area and in methods development. John Hendricks expanded his work with magic square and magic cube particularly with the ornate and embedded varieties that represents new direction in the mathematical field [9].

\section{MAGIC SQUARE CONSTRUCTION}

There is no specific method or limited algorithm to build or constructs all the types of magic square. So the algorithm which works for even squares order will not work for odd order without some additional work or modification, except for the trial and developing computer software by using programming languages. Various methods for constructing magic squares have been evolved through the ages. When considering these methods, it is useful to categorize magic squares in to three classes according to the matrix dimensions by discussing three most common techniques of the construction magic squares: those of an odd order; 
those of a singly even order and the doubly even order technique [10].

\section{A. Magic Squares of Odd Order}

One of the easiest of the three types of magic square where the order $\mathrm{n}$ is of the form $2 \mathrm{~m}+1$, where $\mathrm{m}$ may be any positive integer $(1,2,3$, etc.). The example includes the De la Loubère's method. The matrix dimension will be $3 * 3,5 * 5$, and $7 * 7$ and so on. Odd order magic squares are fairly easily constructed by using any one of these method such as pyramid or serrated method and another method like de la Loubere's or the Staircase method, the Lozenge, or the de Meziriac's method [11].

\section{B. Magic Squares of a Doubly Even Order}

The Doubly even order squares where the order $\mathrm{n}$ is of the form $4 \mathrm{~m}$, such as $(4,8,12,16,32$, etc.). The order of doubly even square can be divided by 2 and 4 . Example includes the Albrecht Dürer's method. The dimension of square matrix will be $4 * 4,8 * 8$, and $12 * 12$ and so on. There is no specific or fixed algorithm for constructing the even-order squares. However, there are some methods can be applicant for building magic squares of even orders [12].

\section{Magic Squares of a Singly Even Order}

Squares where $n$ is of the form $2(2 m+1)=4 n+2$, such as $(2,6,10,14,18,22$, etc.). The order of a singly even square can be divided by 2 but not 4 . The example includes the Philippe de la Hire's method. The matrix dimension will be $6 * 6,10 * 10$, and $14 * 14$ and so on [13].

\section{MAGic SQUARE PROPERTIES}

There are several fascinating features and marvelous properties for the normal magic square which rule and determine the general observations and the nature of the magic square's construction that can be listed below [14]:

- The addition of certain number to each number in the square keeps the square magically.

- The multiplication of certain number by each number in the square keeps the square magically.

- The exchange of two columns or two rows from the center of square equidistantly keeps the square magically.

- The magic square of an even order with interchanged quadrants keeps the square magically.

- The magic square of an odd order with rows interchange and partial quadrants maintains the magic square.

- The fantastic properties for the square matrix makes the square "magic" because it contains a distinct positive integers from $1,2, \ldots, n^{2}$ and the vector sum for all directions is the same.

- The magic square's order determined by the size or dimension of square matrix.

- The probabilities of construction magic square are increases rapidly with the size or order of magic square that involves several forms of magic square except the rotations and reflections cases.

\section{MAGIC CUBE}

Magic cube is an extension to the magic square with three dimensions or more, that contain an arrangement set of sequence integer numbers from $1,2, \ldots n^{3}$. The sum of the entries elements in the rows, columns, and all the diagonals gives the same magic constant of cube. A magic cube construction of order 3 is shown in below Fig. 3 [15].
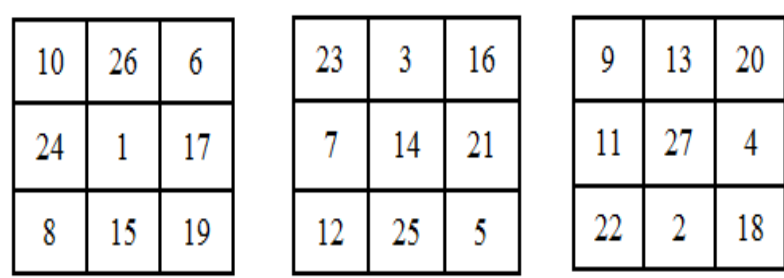

Fig.3. Magic Cube with Three Dimensions

The magic cube likes the magic square from the point of probability construction that increases dramatically with the order of magic cube. The following magic cube in Fig. 4 represents another direction in the cube construction. The starting element in the diagonal cube begins from one corner of the cube that comprises the upper right dimensions to the lower left corner. This is the smallest normal magic cube of $3 \times 3 \times 3$ dimension with sequential numbers from 1 to 27 that are organized in three layers of nine numbers and the magic constant for this cube is sum to 42 [16].

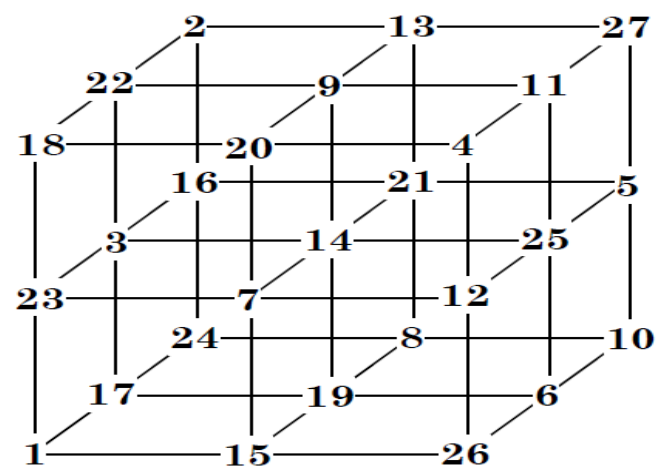

Fig.4. Magic Cube with Sequential Numbers

The Magic Constant (MC) for the normal magic cube (M Cube) can be calculated as follow:

$$
\text { M Cube }=\frac{n\left(n^{3}+1\right)}{2}
$$

The magic constant (MC) for a Tesseract or quadrilateral that will be referred by (MT) formula can be calculated as follow:

$$
M T=\frac{n\left(n^{4}+1\right)}{2}
$$


The generalization of magic constant of $n$-dimensional layout such as hypercube can be calculated as follow:

$$
G=\frac{n\left(n^{n}+1\right)}{2}
$$

Magic cubes are more than playing games with numbers likes the chessboard or Rubik cube, but they substantially depends upon the mathematical rules in their construction. Magic cubes are embedded in several mathematical fields like the number theory, matrices, and combinatorics. There exists an eleven of distinct flat shapes that can be folded-up to construct a shape of cube as stated in the Fig. 5 at the end of paper [17].
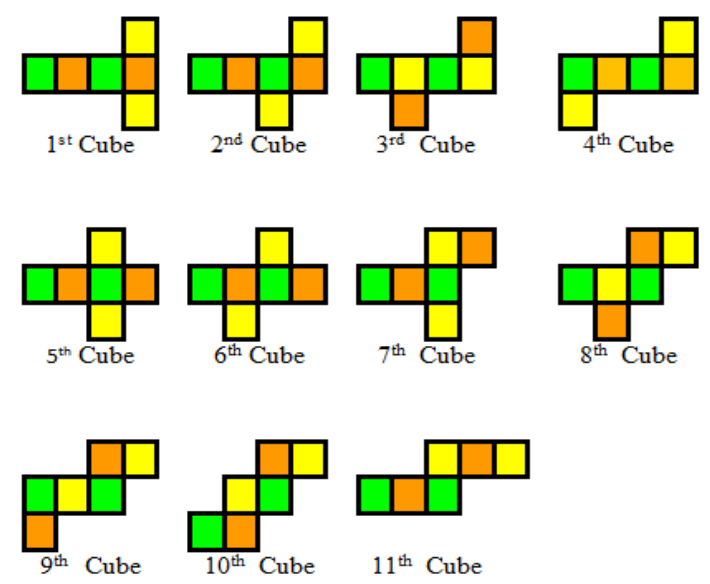

Fig.5. Cubes with Different Shapes

The shapes have been colored with three colors (green, yellow and orange) each pair of opposite sides are colored with same color to constitute a folded cube with six surfaces each two opposite surfaces with the same color. The purpose of theses colors is to keep the arrangement of the magic square properly and to maintain the magical properties as we shall explain in the next sections.

\section{THE PROPOSED METHOD}

The construction of magic cube is the most difficult problem that became interesting to researchers in the mathematical sciences for a long time. So the methods that work for an odd order of magic cube will not work for doubly even or singly even methods and vice versa. The proposed method works for all types of magic cube and with any order and it depends basically on the magic square techniques. The proposed method allows constructing several magic cubes with sequential numbers or with constant differences among the series of numbers. The work for six squares (surfaces) will give one magic cube and the work for twelve squares will constitute two magic cubes and so on, so the work should be multiples of six to introduce several cubes regarding the need or to the task requirements. This means each series of six magic squares can be constructed as a magic cube. The following example explains the core idea for the magic cube construction.

1. Build six separated magic square of any order corresponding to the six surfaces of the cube dimensions as shown in Fig. 6 with order three.

2. Arrange the six surfaces (squares) of the cube by the following way: the first surface should be put opposite to the sixth surface and the second surface opposite to the fifth surface and finally places the third surface opposite to the forth surface with the corresponding colours respectively.

\begin{tabular}{|c|c|c|}
\hline 21 & 0 & 15 \\
\hline 6 & 12 & 18 \\
\hline 9 & 24 & 3 \\
\hline \multicolumn{3}{|c|}{$1^{\text {st }}$ Square }
\end{tabular}

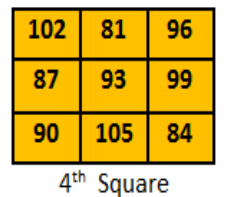

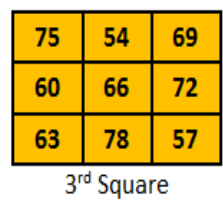

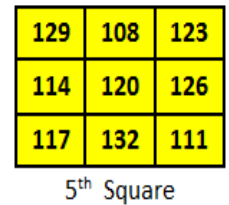

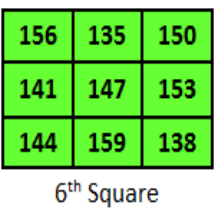

Fig.6. Cube's Dimensions of Order Three

After constructing and coloring the six surfaces (dimensions) for the magic cube as it illustrated in the previous Fig. 6, the magic constant and the magic sum are computed for each square consecutively. The summation for each pairs of analogues colored square will give the same result as shown in Fig. 7, and in Fig. 8, respectively.

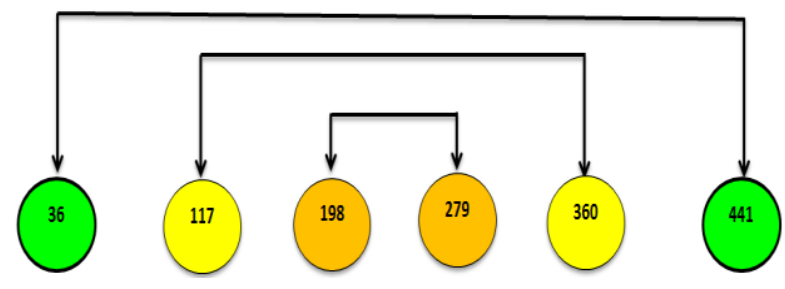

Fig.7. The Magic Constant of the Six Squares

$36+441=\mathbf{4 7 7}, 117+360=\mathbf{4 7 7}, 198+279=\mathbf{4 7 7}$

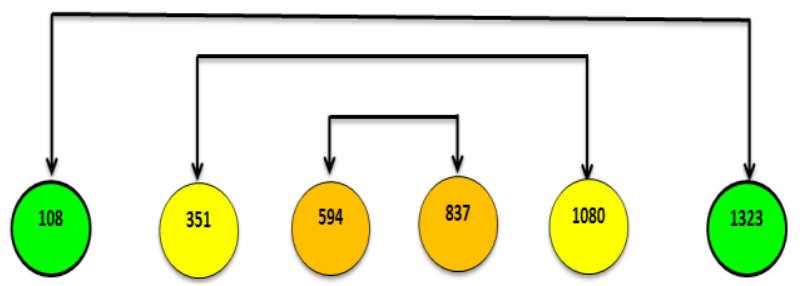

Fig.8. The Magic Sum of the Six Squares

$108+1323=\mathbf{1 4 3 1}, 351+1080=\mathbf{1 4 3 1}, 594+837=\mathbf{1 4 3 1}$

The magic cube in the previous Fig. 6, is of order three with starting value 0 and the constant difference 3 
between each pair of numbers in the $1^{\text {st }}$ Square like the following sequence $(0,3,6,9 \ldots 24)$. The $2^{\text {nd }}$ Square starts and ends with the following arrangement $(27,30,33$, $36 . .51)$ and so on until to the last or the $6^{\text {th }}$ Square of the cube that starts with the number of 135 and ends with 159 .
The numerical values mentioned in Table 1 specify some important properties for the magic sum and magic constant for the magic square that symbolizes the dimensions for the cube.

Table 1. Magic Sum (MS) and Magic Constant (MC) Computations

\begin{tabular}{|l|c|c|c|}
\hline \multicolumn{1}{|c|}{ MC } & MS & MC & MS \\
\hline $441-360=81$ & $1323-080=243$ & $36 \bmod 81=36$ & $108 \bmod 243=108$ \\
$360-279=81$ & $1080-837=243$ & $117 \bmod 81=36$ & $351 \bmod 243=108$ \\
$279-198=81$ & $837-594=243$ & $198 \bmod 81=36$ & $594 \bmod 243=108$ \\
$198-117=81$ & $594-351=243$ & $279 \bmod 81=36$ & $837 \bmod 243=108$ \\
$117-36=81$ & $351-108=243$ & $360 \bmod 81=36$ & $1080 \bmod 243=108$ \\
& & $441 \bmod 81=36$ & $1323 \bmod 243=108$ \\
\hline
\end{tabular}

The following Fig. 9 considers additional completed example of constructing magic cube, which involves six surfaces squares for the cube with doubly even method of order four that contains sequential numbers from 1 to 96.
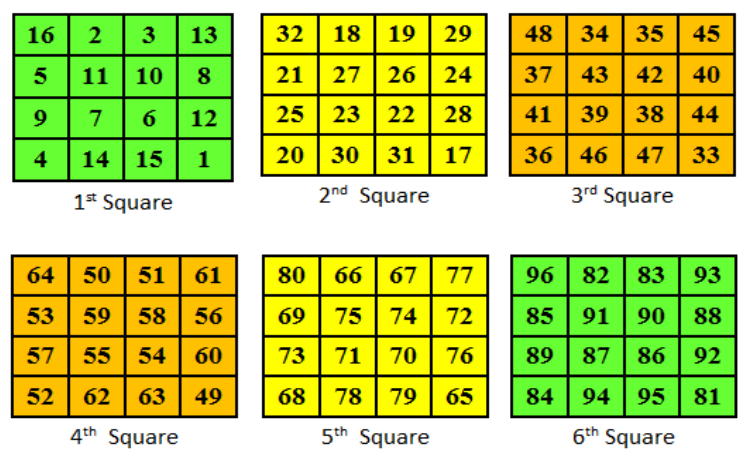

\begin{tabular}{|l|l|l|l|}
\hline 80 & 66 & 67 & 77 \\
\hline 69 & 75 & 74 & 72 \\
\hline 73 & 71 & 70 & 76 \\
\hline 68 & 78 & 79 & 65 \\
\hline \multicolumn{5}{|c|}{$5^{\text {th }}$ Square } \\
\hline
\end{tabular}

\begin{tabular}{|l|l|l|l|}
\hline 96 & 82 & 83 & 93 \\
\hline 85 & 91 & 90 & 88 \\
\hline 89 & 87 & 86 & 92 \\
\hline 84 & 94 & 95 & 81 \\
\hline \multicolumn{4}{|c|}{$6^{\text {th }}$ Square }
\end{tabular}

Fig.9. Cube's Dimensions of Order Four

As it was mentioned earlier in the previous sections that there exist eleven forms of distinct shapes that can be folded-up to construct a cube. The proposed method exploits this feature and fills the squares with different numbers according to the magic square rules to constitute the magic cube. The Fig. 10 at the end of this paper explains the shapes with six dimensions painted with three different colors which construct a cube after they are folded.
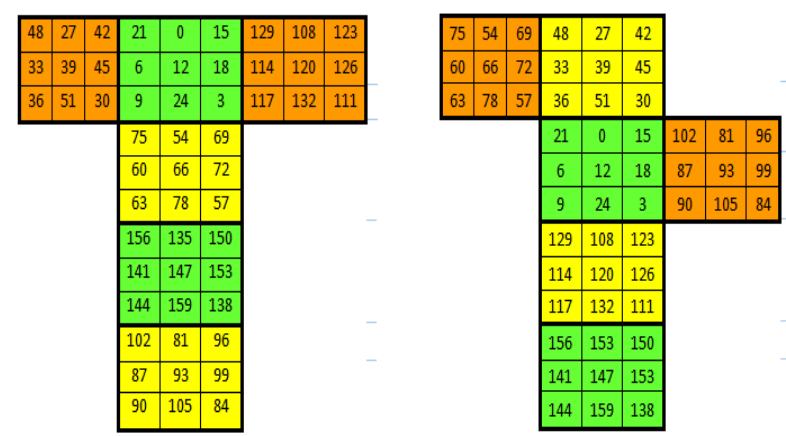
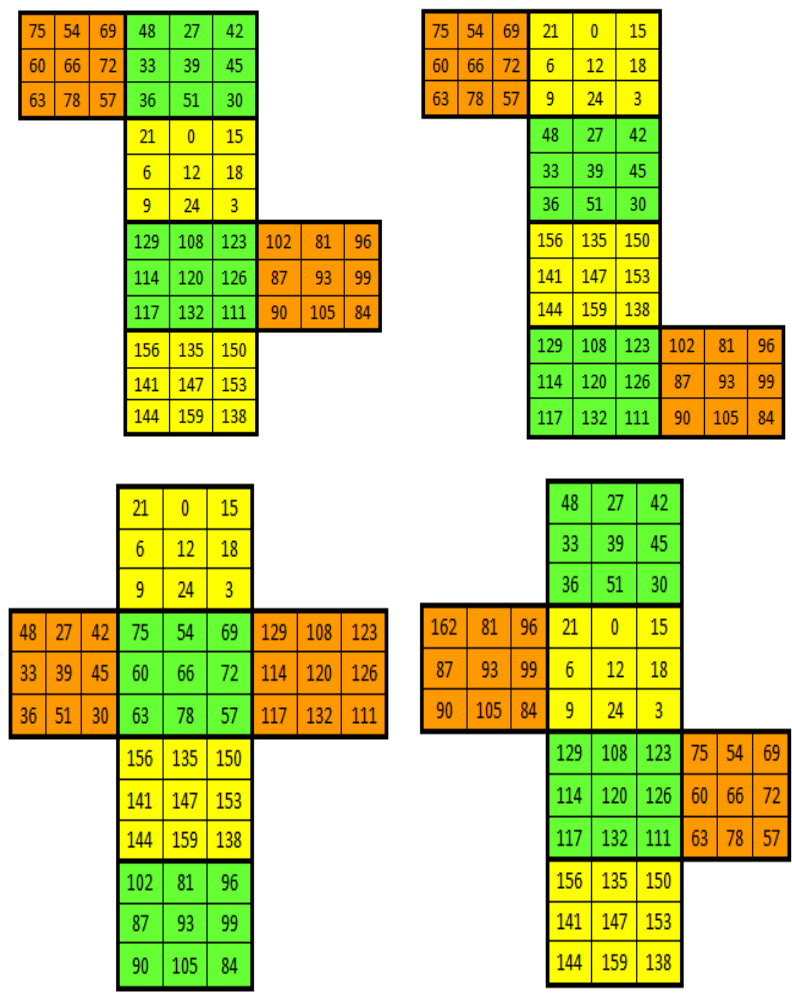

Fig.10. (a) Shapes of Magic Cube
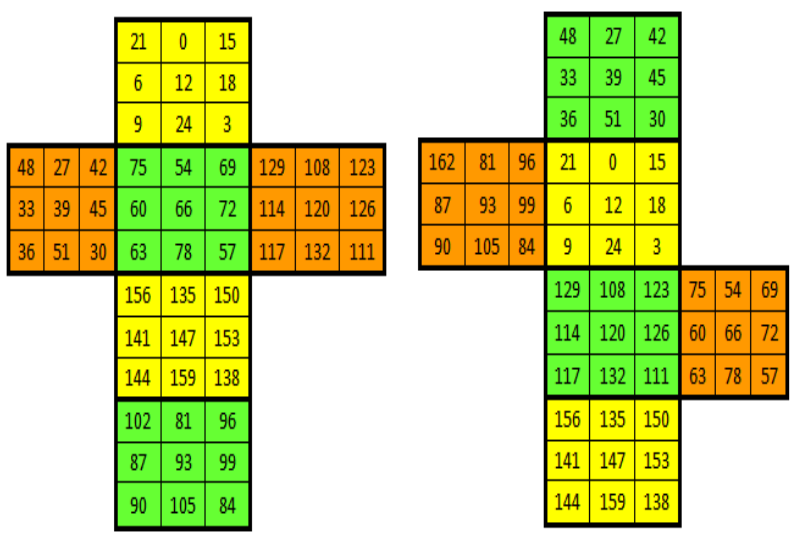

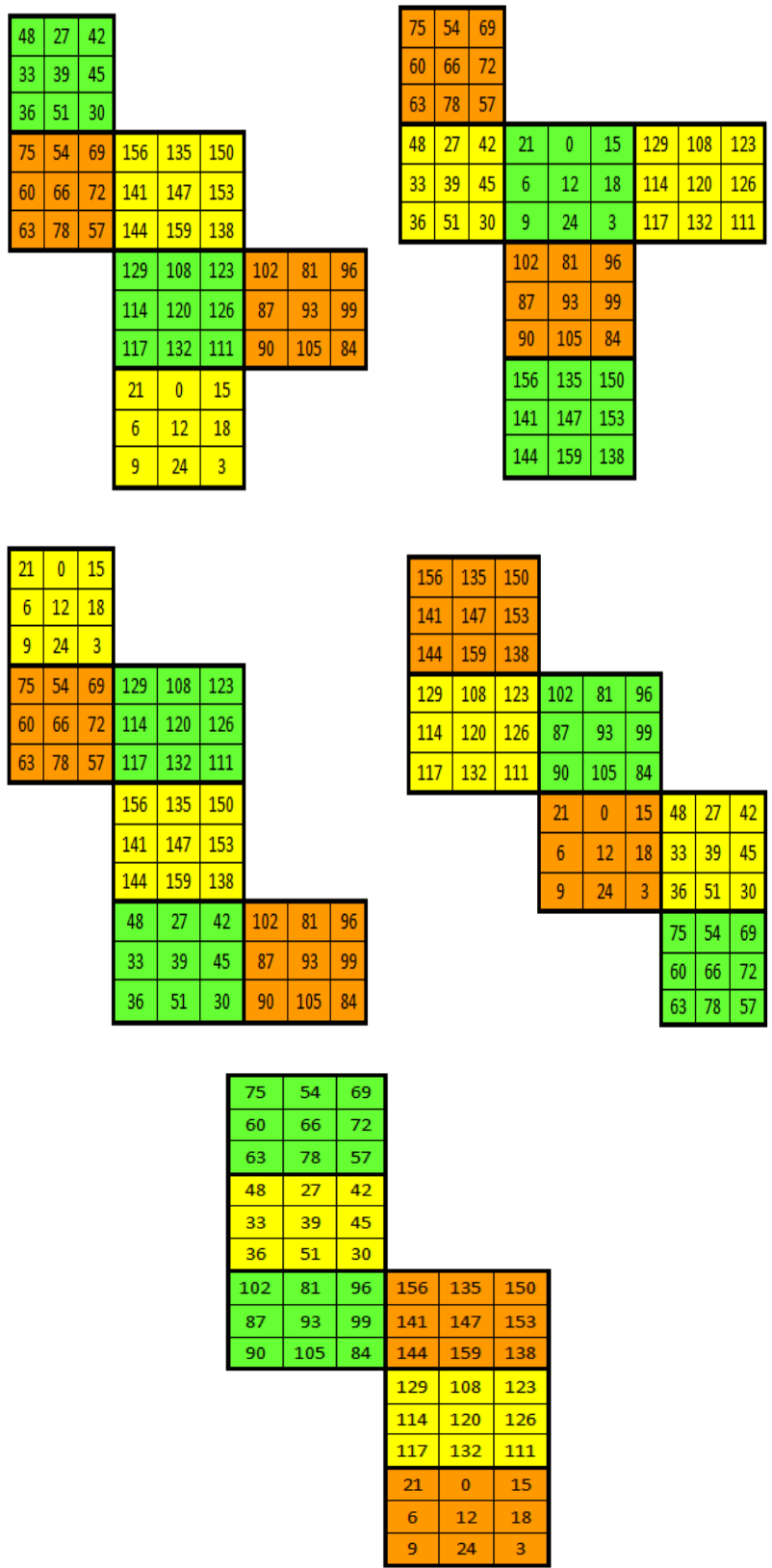

Fig.10. (b) Other Shapes of Magic Cube

\section{THE CRoss Figure of Folded CuBE}

The cross shape which states in Fig. 11 below acts one of the several shapes that construct the magic cube, which is selected to be traversed and tested with vertical and horizontal track for the main diagonals values and the secondary diagonals values, from all directions which will produce the same constant number of (954), as shown in Fig. 12 and Fig. 13 respectively. There is no problem or restricted matter in the selection of any shape from the magic cube's shapes else cross shape, where the cross shape is chosen because it is very easy, simple and clear to figure out the procedures of folding in the magic square surfaces. The cross shape will be partitioned into several basic parts in order to study its properties in detail.

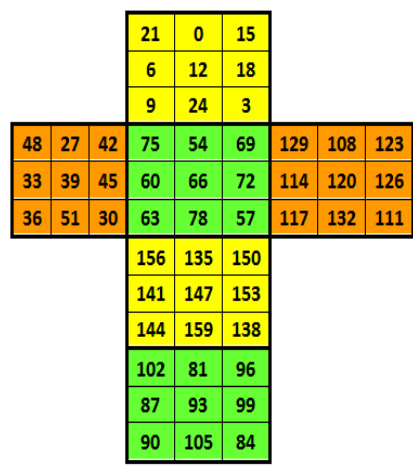

Fig.11. Unfolded Magic Cube

The first part will comprise the two diagonals that comprise the main and secondary diagonals for the matrices of magic cube with circular movement in both directions vertically and horizontally as shown in Fig. 12 and Fig. 13 respectively.

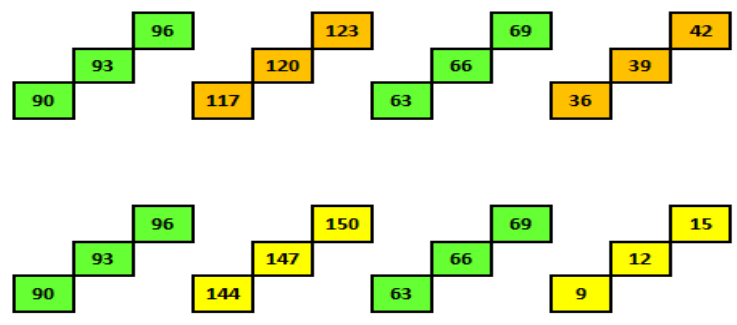

Fig.12. The Vertical and Horizontal Track of Secondary Diagonals

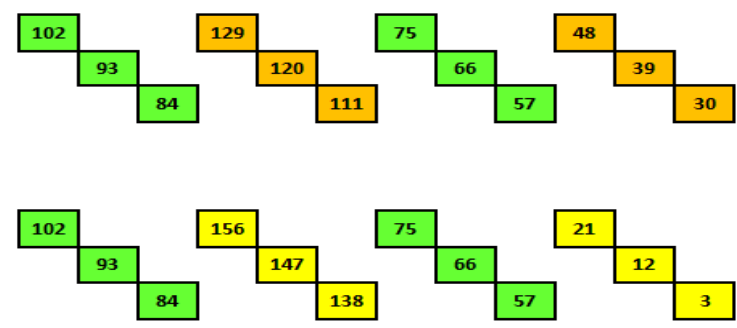

Fig.13. The Vertical and Horizontal Track of Main Diagonals

The second part of the cube will involve the rows and columns of magic cube with circular movement in both directions vertically and horizontally and the traversing process that comprises the tracks of values with rows and columns of the magic cube from all directions. These operations also will produce the same constant number of (954) as it is explained in Fig. 14 and Fig. 15 respectively.

\begin{tabular}{|l|l|l|l|l|l|l|l|l|l|l|l|}
\hline 102 & 81 & 96 & 48 & 27 & 42 & 75 & 54 & 69 & 129 & 108 & 123 \\
\hline 87 & 93 & 99 & 33 & 39 & 45 & 60 & 66 & 72 & 114 & 120 & 126 \\
\hline 90 & 105 & 84 & 36 & 51 & 30 & 63 & 78 & 57 & 117 & 132 & 111 \\
\hline
\end{tabular}

Fig.14. The Vertical Track of Cube's Rows Values

\begin{tabular}{|l|l|l|l|l|l|l|l|l|l|l|l|}
\hline 90 & 87 & 102 & 144 & 141 & 156 & 63 & 60 & 75 & 9 & 6 & 21 \\
\hline 105 & 93 & 81 & 159 & 147 & 135 & 78 & 66 & 54 & 24 & 12 & 0 \\
\hline 84 & 99 & 96 & 138 & 153 & 150 & 57 & 72 & 69 & 3 & 18 & 15 \\
\hline
\end{tabular}

Fig.15. The Horizontal Track of Cube's Columns Values 


\section{ADVANTAGES AND LIMITATIONS}

There are several pros and cons for the construction of the proposed model of the magic cube that can be listed as follows:

\section{A. The Advantages of the Proposed Model of Magic Cube}

- It is an easy, elegant and fast method that requires no substantial efforts in its construction, since it depends upon the tradition magic square methods.

- The proposed method considers a generalized technique to any order of magic cube those of an odd order, even order and doubly even order.

- The proposed method does not require much of thinking or deep understanding in the cube geometry field and does not need long experience in the cube comprehension design or mathematical background.

- The vertical track, horizontal track or diagonals track of values give the same magic constant of cube and the same magic sum of cub that keeps the cube magically.

- The proposed approach work with cube of six dimension or six faces in opposite to the methods those work with three dimensions.

\section{B. The Limitations of the Proposed Model of Magic Cube}

- The construction of the proposed magic cube has no independent clue, since it is based mainly on the magic square techniques.

- The construction of this cube is not based on the specific algorithm of magic square, because it will need different algorithms according to the dimension of square through which it is constructed.

- In spite of the proposed method is easy and does not need the mathematical background, but it requires some knowledge or experience in the magic square methods.

\section{APPliCATIONS OF THE PROPOSED MAGIC CUBE}

Magic cube has fascinating and delightful features and appeals to everyone, but has no specific applications. Recently several published research papers embed the magic square and magic cube and exploited their properties in many aspects and in several scopes such as:

1. Cryptography and Information Security.

2. Public Key and Secret Sharing.

3. Remote Access Control.

4. Applied Mathematics.

5. Number Theory.

6. Determinants and Matrices Field.

7. Coding and error Correctness

\section{CONClusions}

The proposed method is considered the simplest and nearly the fastest method to construct the magic cube since it is based on the traditional magic square methods and can be constructed with any order easily. There is no existence for any real difficulty in the construction of any magic cube with this technique, because it depends upon the folded process for the magic square, and does not need any geometrical measurements. Several studies have been published in the past from different academic researchers about the magic cube, but did not satisfy the objective. Some of these proposed methods were limited for specific order and the other were appropriate just for odd order and the last category included the magic cube only with three or five faces (dimensions). The most obvious conclusions that can be drawn from this paper are the construction of magic cube with six faces and with any order as well as with unspecific or unlimited starting number, which makes the method generalized.

\section{ACKNOWLEDGMENT}

The authors would like to thank Dr. Hazim Hakoosh for his great efforts and they are grateful for his valuable comments and suggestions from the grammatical term.

\section{REFERENCES}

[1] Evel'in Fonseca Cruz and Enguerran Grandchamp, "Heuristic Method to Find Magic Squares", IEEE Computer Society, 15th International Conference on Computational Science and Engineering 2012.

[2] Pickover, C. A.: The Zen of Magic Square, Circles and Stars: An Exhibition of Surprising Structures Across Dimensions, NJ: Princeton University Press, 2002.

[3] B L Kaul, "Generalization of Magic Square (Numerical Logic) $3 \times 3$ and its Multiples $(3 \times 3) \times(3 \times 3)$ ", I.J. Intelligent Systems and Applications, 2013, 01, 90-97

[4] H. D. Heinz \& J. R. Hendricks, "MAGIC SQUARE LEXICON: ILLUSTRATED", Copyright (C) 2000 by Harvey D. Heinz.

[5] B L Kaul and Ramveer Singh Generalization of Magic Square (Numerical Logic) $3 \times 3$ and its Multiples $(3 \times 3) \times$ (3×3), I.J. Intelligent Systems and Applications, 2013, 01, 90-97.

[6] Tomba I and Shibiraj N, "Improved Technique for Constructing Doubly-even Magic Squares using Basic Latin Squares", International Journal of Scientific and Research Publications, Volume 3, Issue 6, June 2013.

[7] John Hendricks, "The Diagonal Rule for Magic Cubes of Odd Order," Journal of Recreational Mathematics 20(4):192-195 (1988).

[8] William H. Benson and Oswald Jacoby, "MAGIC CUBES New Recreations", Dover Publications, Inc., in 1981.

[9] C.A. Pickover, The Zen of magic squares, circles, and stars, Princeton University Press, Princeton, NJ, 2002.

[10] D.I. George, J.Sai Geetha and K.Mani, "Add-on Security Level for Public Key Cryptosystem using Magic Rectangle with Column/Row Shifting", International Journal of Computer Applications (0975 - 8887) Volume 96-No.14, pp. 38-43, June 2014. 
[11] Nitin Pandey, D.B.Ojha, "SECURE COMMUNICATION SCHEME WITH MAGIC SQUARE", Volume 3, No. 12, pp. 12-14 December 2012.

[12] Gopinanath Ganapathy, and K. Mani, "Add-On Security Model for Public-Key Cryptosystem Based on Magic Square Implementation", Proceedings of the World Congress on Engineering and Computer Science, San Francisco, USA, Vol I, 2009.

[13] Ronald P. Nordgren, "NEW CONSTRUCTIONS FOR SPECIAL MAGIC SQUARES", International Journal of Pure and Applied Mathematics, Volume 78 No. 2, pp 133154, Academic Publications, Ltd.2012.

[14] Adam Rogers, and Peter Loly, "The Inertial Properties of Magic Squares and Cubes", Nov. 2004, pp. 1-3.

[15] Tao Xie and Lishan Kang, "An Evolutionary Algorithm for Magic Squares", IEEE, 2003.

[16] FRANK J. SWETZ, "Legacy of the Luoshu", The 4,000 Year Search for the Meaning of the Magic Square of Order Three, A K Peters, Ltd. Wellesley, Massachusetts, 2008.

[17] Andrews, W. S. Magic Squares and Cubes. Chicago: SECOND EDITION. REVISED AND ENLARGED Open Court Publishing, 1917.

\section{Authors' Profiles}

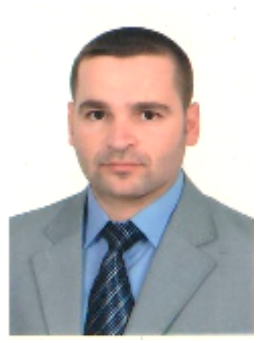

Omar A. Dawood was born in Habanyah, Anbar, Iraq (1986), now he lives in Ramadi, Anbar. He obtained B.Sc. (2008), M.Sc. (2011) in Computer Science from the College of Computer, Anbar University, Iraq. He was ranking the first during his B.Sc. and M.Sc. studies. He is a teaching staff member in the English Department in College of Education for Humanities, Anbar University, and currently he is a Ph.D. student at the Technology University- Baghdad. His research interests are: Data and Network Security, Coding, Number Theory and Cryptography.

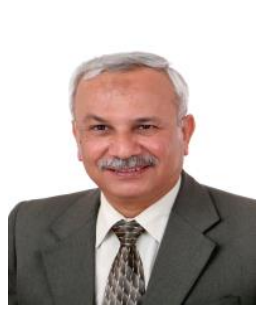

Prof. Abdul Monem S. Rahma Ph.D Awarded his M.Sc. from Brunel University and his Ph.D. from Loughborough University of technology United Kingdom in 1982, 1984 respectively. He taught at Baghdad university Department of Computer Science and the Military Collage of Engineering, Computer Engineering Department from 1986 till 2003. He holds the position of Dean Asst. of the scientific affairs and works as a professor at the University of Technology computer Science Department. He published 88 Papers, 4 Books in the field of computer science, supervised $28 \mathrm{Ph} . \mathrm{D}$. and $57 \mathrm{M} . \mathrm{Sc}$. students. His research interests include Computer Graphics Image Processing, Biometrics and Computer Security. And he has attended and submitted in many scientific global conferences in Iraq and many other countries. From 2013 to Jan. 2015 he holds the position of Dean of the Computer Science College at the University of Technology.

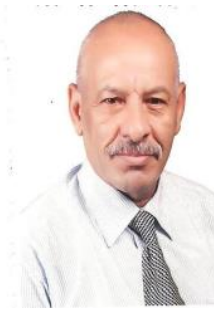

Abdul Mohssen J. Abdul Hossen is an Associate Professor of Applied mathematics, Computer Science Department, University of Technology, where he teaches undergraduate and graduate courses in Mathematics. Dr. Abdul Hossen received the B.Sc. in Mathematics from Mustansiriyah University, Iraq 1977, the M.Sc. degree in Applied Mathematics from Bagdad University, Iraq. in1980, the Ph.D. in Applied Mathematics from University of Technology, Iraq, 2005. He is a member of the IEEE system, and Member of the Editorial Journal.

How to cite this paper: Omar A. Dawood, Abdul Monem S. Rahma, Abdul Mohsen J. Abdul Hossen, "Generalized Method for Constructing Magic Cube by Folded Magic Squares", International Journal of Intelligent Systems and Applications (IJISA), Vol.8, No.1, pp.1-8, 2016. DOI: 10.5815/ijisa.2016.01.01 